\title{
A flexible and fully integrated system for amplification, detection and genotyping of genomic DNA targets based on microfiluidic oligonucleotide arrays
}

\author{
Daniel Summerer ${ }^{1,3}$, Dona Hevroni ${ }^{2,3}$, Amit Jain ${ }^{2,3}$, Olga Oldenburger ${ }^{1}$, \\ Jefferson Parker ${ }^{2}$, Anthony Caruso ${ }^{2}$, Cord F. Stähler ${ }^{1}$, Peer F. Stähler ${ }^{1}$ and \\ Markus Beier ${ }^{1}$
}

\footnotetext{
${ }^{1}$ febit biomed gmbh, Im Neuenheimer Feld 519, 69120 Heidelberg, Germany

${ }^{2}$ febit Inc., 99 Hayden Avenue, Lexington, MA 02421, USA
}

A strategy allowing for amplification, detection and genotyping of different genomic DNA targets in a single reaction container is described. The method makes use of primer-directed solution-phase amplification with integrated labeling in a closed, microfluidic oligonucleotide array. Selective array probes allow for subsequent detection and genotyping of generated amplicons by hybridization. The array contains up to 15,624 programmable features that can be designed, de novo synthesized and tested within 24 hours using an automated benchtop microarray synthesizer. This enables rapid prototyping and adaptation of the system to newly emerging targets such as pathogenic bacterial or viral subtypes. The system was evaluated by amplifying and detecting different loci of viral (HPV), bacterial (Bacillus sp.) and eukaryotic (human) genomes. Multiplex PCR and semi-quantitative detection with excellent detection limits of $<100$ target copies is hereby demonstrated. The high automation grade of the system reduces contamination risk and workload and should enhance safety and reproducibility.

\section{Introduction}

The ever increasing understanding of organism complex nucleic acid repertoire such as genome structure and stability, microbial diversity or transcriptional dynamics has called for highly parallel detection, identification and quantification of nucleic acids. Owing to its excellent sensitivity and accuracy, PCR has hereby been a central target for the development of highly multiplexed assay technologies.

To increase throughput of PCR assays, several strategies have been followed. One strategy is the combination of multiple homogenous PCR systems using several specific or degenerate primer pairs within one reaction vessel. Target detection is then often achieved by using fluorescent signaling probes, such as labeled oligonucleotides that undergo changes in fluorescence behavior owing to nucleolytic cleavage or conformational changes during

Corresponding author: Summerer, D. (dantel.summerer@febit.de)

${ }^{3}$ These authors contributed equally to this work.
PCR product formation [1-5]. Many of these methods are very mature, allow for quantitative real-time analysis of samples for multiple targets and have found widespread application in research and molecular diagnostics. However, a limitation of homogenous multiplex PCR systems with fluorescent signaling has so far been the relatively low multiplexing grade owing to spectral overlap and resulting cross-talk of fluorophores.

A second strategy for increased throughput that circumvents cross-talk by spatial separation is the parallelization of individual, homogenous PCR setups with limited or no primer-pair multiplexing within single reactions. However, scaling PCR to analyze larger numbers of targets and samples simultaneously is limited by the logistics and cost of the assay when performed in traditional multiwell-plate formats. Consequently, recent developments have focused on the miniaturization of individual PCR reactions leading to parallel methods with high to very high throughput [6-14]. Although multiplexing grade in terms of amplification targets within a given sample can be increased by higher parallelization 
of individual reactions, this approach is limited. Partitioning of a sample has to be compensated by increased sensitivity of individual assays. Moreover, this strategy is inapplicable in cases where the required assay numbers for a sample exceed copy numbers of individual targets due to overdilution. However, assays in molecular diagnostics may often require the detection of low abundance nucleic acids in the range of $<100$ copies per sample, which imposes an intrinsic limit to the approach. Hence, there is increasing demand for methods that combine high multiplexing grade of targets without sample partitioning during PCR. One attractive option is to apply heterogenous detection systems to multiplex PCRs conducted in single vessels. This allows for spatial separation of detection events and individual readout without signal crosstalk as in homogenous detection systems. This strategy has, for example, been used in a method that applies beads with individual, target-specific receptors such as oligonucleotide probes that are simultaneously added to an amplicon mixture for binding. Up to 100 different bead types can thereby be optically identified by fluidic separation and color coding and bound PCR products quantified by fluorescence $[15,16]$.

Even higher multiplexing grades can be achieved by the use of oligonucleotide arrays that can simultaneously detect hundreds of thousands or millions of different nucleic acid sequences in parallel [17]. Numerous microarray-based assays have been described for the detection of different target types such as viruses, bacterial pathogens or human genetic variants [18-23]. However, workflows of such methods have been rather labor- and time-intensive with partially separated amplification, labeling, microarray hybridization, washing and detection, often involving purification and individual hardware for the various processing steps.

Here, we describe a method using only one processing station and a single, microfluidic oligonucleotide array that serves as a low volume compartment for all steps of a nucleic acid detection and typing process. This includes amplification and labeling of nucleic acid targets, array hybridization, washing, fluorescent staining and detection of individual PCR products. The method is evaluated with viral, bacterial and human nucleic acid targets in multiplexing mode and a detection limit of $<100$ copies is demonstrated. By using a fully automated platform for de novo array synthesis, probe content is highly flexible with a prototyping iteration cycle of probe design, microarray synthesis, experimental testing and microarray redesign of less than 24 hours. This allows the rapid development of novel assay formats to adapt the system to novel target sequences such as emerging viral or bacterial pathogenic subtypes.

\section{Materials and methods \\ DNA samples and oligonucleotides}

Plasmid containing the entire genome of HPV 6b (ATCC-45150D) was obtained from LGC Promochem. Bacterial genomic DNA was obtained from ATCC. Used species were $B$. cereus (ATCC 14579), $B$. subtilis str. 168 (ATCC 23857) and B. thuringiensis ser. israelensis (ATCC 35646). Oligonucleotides were purchased from Sigma Genosys.

\section{Amplification protocols}

For HPV PCR experiments, varying copy numbers of pHPV6b were amplified using Absolute Mastermix (ABgene) in the presence of
$100 \mu \mathrm{M}$ Biotin-16-dUTP and $0.5 \mu \mathrm{M}$ primer pool MY09 (CGTCCMARRGGAWACTGATC), $0.5 \mu \mathrm{M}$ primer pool MY11 (GCMCAGGGWCATAAYAATGG), varying amounts of human genomic DNA (Promega) and/or $0.25 \mu \mathrm{M}$ primer $\beta$-Glob_fwd (CAACTTCATCCACGTTCACC) and $0.25 \mu \mathrm{M}$ primer $\beta$-Glob_rev (GAAGAGCCAAGGACAGGTA), PCRs from a single mastermix were conducted as control in parallel in tubes using a Mastercycler (Eppendorf) and in a microfluidic Geniom Biochip using an Amplispeed microarray slide thermocycler (Advalytix) or a Geniom RT Analyzer instrument. Cycling conditions were as follows: $15 \mathrm{~min} 95^{\circ} \mathrm{C}$, then 10 times: $\left(1 \mathrm{~min} 95^{\circ} \mathrm{C}, 1.5 \mathrm{~min} 57^{\circ} \mathrm{C}, 1 \mathrm{~min}\right.$ $72^{\circ} \mathrm{C}$ ), then 25 times: $\left(1 \mathrm{~min} 95^{\circ} \mathrm{C}, 1 \mathrm{~min} 55^{\circ} \mathrm{C}, 1 \mathrm{~min} 72^{\circ} \mathrm{C}\right)$, then $5 \mathrm{~min} 72^{\circ} \mathrm{C}$. Product mixtures were either analyzed by agarose gel electrophoresis or immediately hybridized in microfluidic channels for reactions carried out in a Geniom biochip (see below). After PCR establishment, identities of all PCR products were confirmed by Sanger sequencing.

6-Plex PCRs targeting four Bacillus strains, HPV $6 \mathrm{~b}$ and human $\beta$-globin contained the same concentrations of primers MY09, MY11, $\beta$-Glob_fwd and $\beta-$ Glob_rev as well as $0.5 \mu \mathrm{M}$ of each of the primers B.cereus_446_F (CCTACTATAATCCATGCA), B.cereus_446_R (GGAGAAGATAGAATTGCT), B.Sub_395_F (CCTTCTATTTCTAACGCA), B.Sub_395_R (CGATAATCATTGATCCGT), B.Thu.Isr_407_F (CCATTCATGATAACTGCT), B.Thu.Isr_407_R (GGTACCGTAATTATTGGA). Reactions further contained $1 \times$ ThermoStart buffer (Abgene), $500 \mu \mathrm{M}$ of each dATP, dGTP and dCTP, $125 \mu \mathrm{M}$ TTP, $18.75 \mu \mathrm{M}$ Biotin-16-dUTP, $0.03125 \mathrm{U} / \mu \mathrm{L}$ ThermoStart DNA polymerase (Abgene) and varying amounts of different templates as specified in the 'Results' section. Cycling conditions were as described above. Product mixtures were either analyzed by agarose gel electrophoresis or immediately hybridized in microfluidic channels for reactions carried out in a Geniom biochip (see below).

\section{Microarray design and synthesis}

Light-activated in situ oligonucleotide synthesis was performed essentially as described [24] using a digital micromirror device (DMD, Texas Instruments). This allows for light-directed activation on a microfluidic array consisting of a glass-silicon-glass sandwich within the Geniom instrument (febit biomed). Depending on the number of DMD micromirrors used for one feature and for the spacing between features, each chip consists of eight arrays with $6776(2 \times 2$ mirrors for each feature with 1 mirror spacing) or 15,624 ( 1 mirror for each feature with 1 mirror spacing) individual features.

For selective detection, 21 mer tiling probes for the targeted PCR products of $\beta-$ globin ( 248 probes), HPV $6 \mathrm{~b}$ ( 880 probes, including surrounding region of target sequence) were designed with a $1 \mathrm{bp}$ resolution to analyze the complete sequences for the specific regions. For further array designs after initial experimental validation of sensitivity and selectivity of probes, a 6776-feature setup was used.

Bacillus detection probes were designed against forward and reverse strands of amplicon regions with a target size of 25 bp using a $5 \mathrm{bp}$ tiling offset. Probes were further selected to eliminate ambiguous bases, runs of four or more identical bases (polyN) and to maximize 5'-terminal uniqueness across the target genomes. Each experimental amplicon generated 60-70 probes per strand and PCR product target. 


\section{Microarray hybridization, detection and data analysis}

For experiments using purified, labeled PCR products, samples were dissolved in febit hybridization mix-1, including febit control oligo mix, heated to $95^{\circ} \mathrm{C}$ for $5 \mathrm{~min}$ and placed on ice. Geniom biochip was denatured by washing with water at $80^{\circ} \mathrm{C}$ and incubated with febit prehybridization buffer for $15 \mathrm{~min}$ at room temperature. Buffer was removed and sample was injected into microfluidic arrays and incubated for four hours at $45^{\circ} \mathrm{C}$. Samples were removed and the biochip was automatically washed consecutively with $6 \times \mathrm{SSPE}$ at room temperature and $0.5 \times \mathrm{SSPE}$ at $45^{\circ} \mathrm{C}$ within the Geniom device. Streptavidin-(R)-phycoerythrin (SAPE, Invitrogen) in $6 \times$ SSPE was injected and the biochip was incubated for $15 \mathrm{~min}$ at room temperature and then washed with $6 \times$ SSPE. Fluorescence image was acquired using the integrated detection system of the Geniom device.

For experiments using integrated hybridization of PCR product mixes, the biochip was directly used for PCR without denaturation or incubation with febit prehybridization buffer. PCRs were conducted using the Amplispeed microarray slide thermocycler (Advalytix) or a Geniom RT analyzer instrument. PCR mastermix as described above was injected into microfluidic channels and biochip was subjected to the PCR program. Chip was denatured for 5 min at $95^{\circ} \mathrm{C}$ immediately after the PCR reaction, cooled to $45^{\circ} \mathrm{C}$ and incubated for $16 \mathrm{~h}$. Afterwards the target solution was removed from arrays and analyzed by electrophoresis on a $2.5 \%$ agarose gel. Biochip was washed and stained as described above. After the first wash step after SAPE-incubation, a protocol for signal amplification was performed. For 6-plex PCRs, incubation with an antibody-solution $(1 \times \mathrm{MES}, 0.925 \mathrm{M} \mathrm{NaCl}, 0.05 \%$ Tween- $20,1 \mathrm{mg} /$ $\mathrm{ml} \mathrm{BSA)}$ containing multi-biotinylated anti-streptavidin antibody (Vector Laboratories; BA-0500, 1:167 diluted) and goat IgG (Sigma; I5256, 1:100 diluted) as second antibody was conducted. After a second incubation with SAPE and washing, amplified fluorescence was detected. For PCRs targeting HPV $6 \mathrm{~b}$ and human $\beta$-globin only, signal amplification was conducted using the Anti-Biotin Oyster 550 (900) signal amplifier antibody (Genisphere) at $10 \mathrm{ng} /$ $\mu \mathrm{L}$ under the conditions described above. For the analysis of fully integrated microarray experiments, raw fluorescence intensities were recorded, medians of probe replicates were calculated and medians of background features (consisting of a single $T$ residue) were subtracted.

\section{Results and discussion}

We aimed at developing an integrated system for multiplex detection and subtyping of various DNA targets using a single reaction vessel with a simple and automatable workflow. The established approach makes use of the closed, microfluidic Geniom biochip that contains eight individual microchannels with a volume of $\sim 3 \mu \mathrm{L}$ each presenting an array of 6776 or 15,624 DNA capture probe features on its inner surface. The overall workflow is outlined in Fig. 1. A purified genomic DNA sample is mixed with a PCR mastermix containing all components necessary for efficient amplification of targeted loci including biotin-16-dUTP for integrated random labeling. The mixture is injected into the chip and

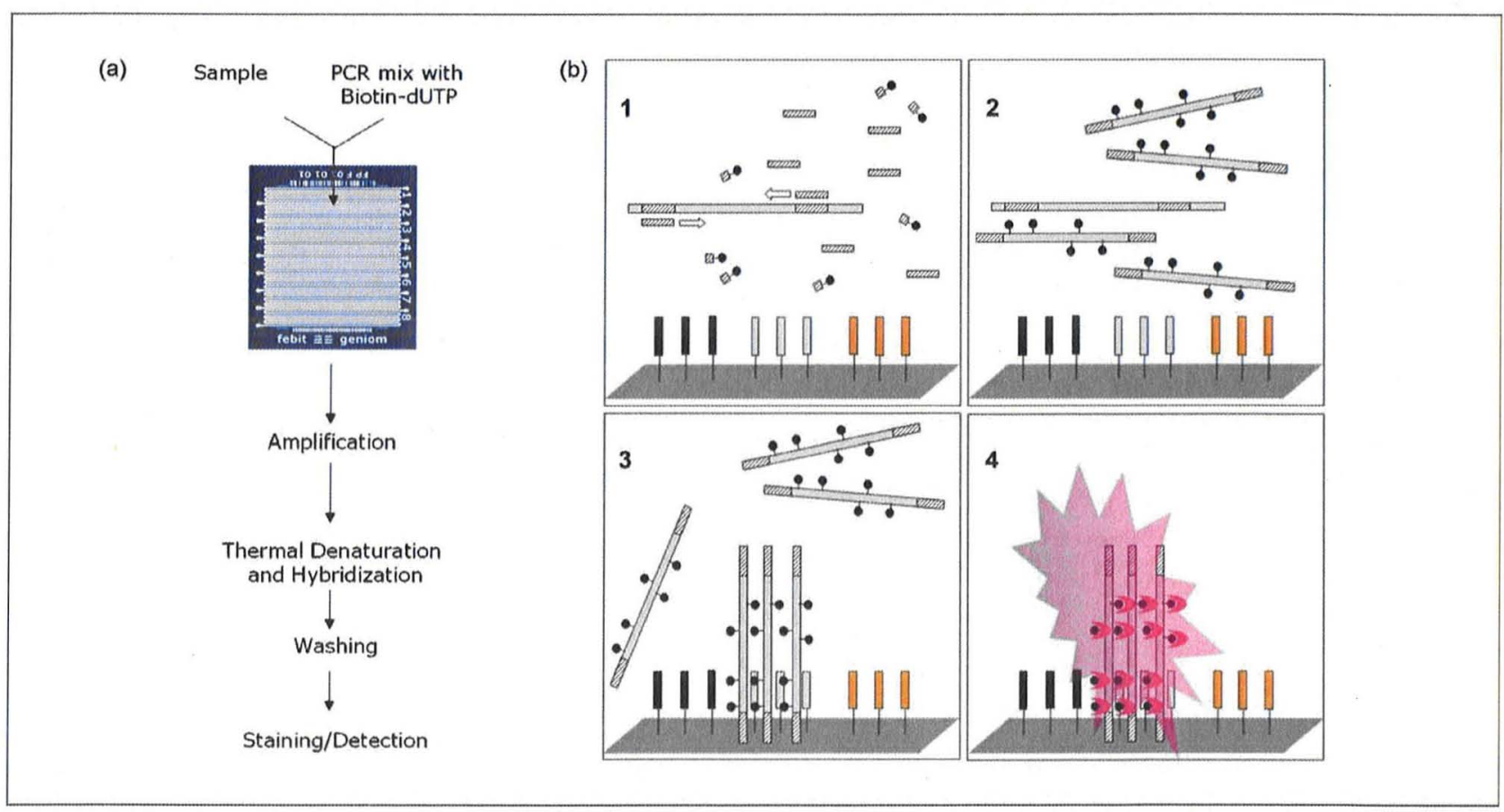

\section{FIGURE 1}

Overview of fully integrated amplification, detection and typing of genomic DNA targets. (a) General workflow. Purified nucleic acid sample containing target nucleic acid is combined with PCR mastermix. Mixture is injected into a microchannel of a Geniom biochip containing selective DNA probes for binding of formed PCR products. Amplification protocol including integrated labeling, hybridization, washing, fluorescent staining and detection is automatically conducted in the employed processing platform. (b) Scheme of in-chip process. Sample DNA molecules are amplified in the presence of biotin-16-dUTP (1) leading to randomly biotinylated PCR products (2). Products are hybridized to selective DNA capture probes and non-or weakly bound DNA is washed away (3). Chip is incubated with Streptavidin-(R)-phycoerythrin, washed and fluorescence is recorded (4). 
amplification is performed within the chip followed by a thermal denaturation and a hybridization step at $45^{\circ} \mathrm{C}$ for selective binding of the probes. After stringent washing, the chip is incubated with a fluorescent streptavidin conjugate (Streptavidin-(R)-phycoerythrin, SAPE), washed and an antibody-based signal amplification process is conducted. Fluorescence is recorded and analysis reveals the presence of individual amplicons.

For the detection of viral genomes, a generic PCR for the amplification of an $\mathrm{L} 1$ region fragment of various human papillomavirus (HPV) subtypes based on the known degenerate primer system MY09/11 was established [25]. The MY09/11 primer set is especially well suited for subtyping using high density DNA microarrays, because PCR products have sufficient size to allow for the design of probes against multiple L1 loci, thereby enhancing reliability of subtype discrimination. Other generic primer systems like the GP5+/GP6+ or SPF mixes yield much smaller products and therefore do not sufficiently match the potential of microarray probe content [26-28]. To verify the presence of human genomic DNA within a sample, PCRs were performed as duplex PCRs containing a second primer pair targeting the human $\beta$-globin gene, which is widely used as positive control marker in human detection assays. Additionally, a multiplex PCR represents a more challenging test-case for the microchannel environment.

For subtyping of PCR products, capture probes were designed for the $\beta$-globin control product and the L1 PCR product of primer pair MY09/11. 21mer tiling probes with a resolution of $1 \mathrm{bp}$ were designed to cover the whole region of all PCR products and a prototype array with a 15,624 feature density was synthesized. In that way, a maximal number of probes per sequence can be experimentally validated by the hybridization of individual PCR products from which a fraction with desired sensitivities and selectivities can be chosen for the design of an optimized subtyping array. Purified, biotinylated PCR products of HPV $6 \mathrm{~b}$ and $\beta$ globin were individually hybridized to two arrays containing all designed probes and relative binding efficiencies as well as crosshybridization tendencies were determined. All probes exhibiting a median discrimination of the noncognate PCR product of $>6$ were used for the design of a second generation subtyping array. This resulted in 168 and 78 probes specific for $\beta$-globin and HPV $6 \mathrm{~b}$, respectively.

This selection was further evaluated in a second round of crosshybridization experiments for the selection of a minimal number of highly specific probes with a 6776 probe array. Overall, 8 probes for HPV $6 \mathrm{~b}$ and 3 probes for $\beta$-globin were used for the final array design. Using 8 probes per target thereby results in a theoretical multiplexing level of $>260$ or $>600$ targets when array densities of 6776 or 15,624 features/array are used and each probe is included in 3 replicates.

PCRs targeting HPV and the human $\beta$-globin gene were conducted in the microchannels of a Geniom biochip with a volume of $\sim 3 \mu \mathrm{L}$ per channel using the Geniom RT Analyzer as processing station. This platform facilitates the workflow of the in chip PCR by featuring integrated PCR temperature cycling, hybridization, washing routines, fluorescence staining and detection. Plasmids containing genomes of HPV subtype $6 \mathrm{~b}$ were spiked in varying concentrations into a background of human genomic DNA. This allows for controlled titration of virus copies in a typical complexity of a patient sample.
As a first test, PCR product formation was analyzed by agarose gel analysis after the removal of the PCR mixture from the channels to assess product purity and detection limit with a standard technique. In singleplex mode, of HPV L1 PCR product was clearly visible with a detection limit of $\sim 620$ copies per $3 \mu \mathrm{L}$. PCR reaction within a background of $0.5 \mathrm{ng}$ human genomic DNA per reaction with good reproducibility (Fig. 2).

When targeting $\beta$-globin in a singleplex PCR, product formation was visible when starting with $0.25 \mathrm{ng}$ ( $\sim 75$ genome copies) of human genomic DNA. Moreover, both the $\beta$-globin and HPV L1 products could be detected by agarose gel electrophoresis when using 310 copies of HPV $6 \mathrm{~b}$ and $0.25 \mathrm{ng}$ human genomic DNA as starting amounts in multiplex PCR mode (Fig. 2). This demonstrates that the employed microchannels can be used as PCR reaction containers that allow for excellent sensitivity. Importantly, these results were obtained using previously known standard PCR primer systems and standard reaction conditions without special adaptation of the applied conditions to the microchannels.

Next, PCR product typing performance of the microarray was tested in the fully integrated workflow with PCR conducted in the microchannels. Chips used for multiplex PCRs containing different starting amounts of human gDNA and HPV $6 \mathrm{~b}$ with a nontemplate control PCR were conducted with integrated hybridization, washing, staining and detection. Fluorescence data of probe features are shown in Fig. 3. No significant fluorescence was observed in negative controls for HPV $6 \mathrm{~b}$-specific probes, whereas

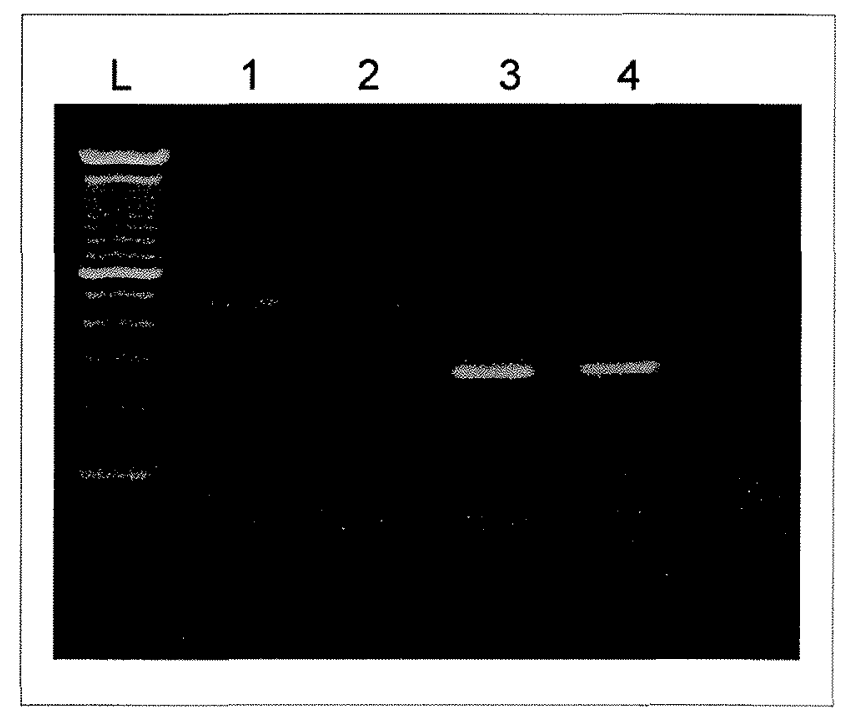

\section{FIGURE 2}

Multiplex PCR for generic amplification of HPV and specific control amplification of $\beta$-globin from samples containing human genomic DNA and HPV genomic DNA. PCR was conducted using the primer pool MYo9/11 for generic amplification of the HPV L1 region, specific primers for human $\beta$ globin and biotin-16-dUTP for integrated labeling. All PCRs were performed in the microfluidic channels of a Geniom biochip. L: Ladder. 1: PCR conducted with 620 copies HPV $6 \mathrm{~b}$ and $0.5 \mathrm{ng}$ human gDNA as template with primers targeting HPV only. 2: Replicate PCR of lane 1. 3: PCR conducted with 310 copies HPV $6 \mathrm{~b}$ and $0.25 \mathrm{ng}$ human $\mathrm{gDNA}$ as template with primers targeting HPV and human $\beta$-globin. 4: PCR conducted with 310 copies HPV $6 b$ and $0.25 \mathrm{ng}$ human gDNA as template with primers targeting human $\beta-g l o b i n$ only. 5: negative control. 
some background fluorescence was visible for the $\beta$-globin probes in the absence of template. However, observed fluorescence for all tested probes clearly exhibited dependence on starting amount of template for both targets. This allows a semi-quantitative analysis of target nucleic acids and could be employed for the determination of viral load and amount of host cell material within a patient sample. Importantly, the detection limit using fluorescence of probe-bound amplicons is lower than the limit obtained for agarose gel electrophoresis and allows the detection of $\sim 100$ copies of HPV $6 \mathrm{~b}, \sim 75$ human genome copies and a viral load of $1.3 \mathrm{HPV} 6 \mathrm{~b}$ copies per human genome copy.

To test further targets and more demanding PCR complexities, a 6-plex PCR system was next established. Four primer pairs targeting individual loci on three different Bacillus strains (Bacillus cereus, Bacillus subtilis and Bacillus thuringiensis ser. israelensis) were designed for maximal specificity between these strains. Primer pairs were individually tested in PCR tubes against their respective target genomic DNA (data not shown). For a 6-plex PCR, the three primer pairs were used in combination with primers specific for HPV $6 \mathrm{~b}$ and $\beta$-globin. PCRs targeting $B$. cereus (3000 genome copies/array), B. subtilis (3000 copies) and B. thuringiensis ser. israelensis (2300 copies) in the presence or absence of human genomic DNA (1 ng/array) were conducted in parallel using a regular PCR tube or the microchannels of a biochip as reaction container. All microchannels contained identical sets of capture probes specific for the targeted amplicons. PCR products were formed in both the presence and absence of human genomic DNA with no clear resolution of Bacillus-related products, presumably owing to similar amplicon lengths (Fig. 4a). No significant formation of byproducts was observed and no product was present in negative control PCR without genomic DNA. PCRs carried out in microchannels with integrated hybridization and fluorescence imaging afforded a collection of capture probes with sufficient intensity for further analysis (Fig. 4b). In contrast to agarose gel electrophoresis, the presence of all three Bacillus-related products was indicated by fluorescence signals in positive reactions (Fig. $4 \mathrm{~b}$, 1-2), whereas no significant fluorescence was observed for most probes in the negative control PCR (Fig. 4b,3). PCR reactions in the absence of human genomic DNA were carried out in duplicate using two arrays and individual capture probes within the two

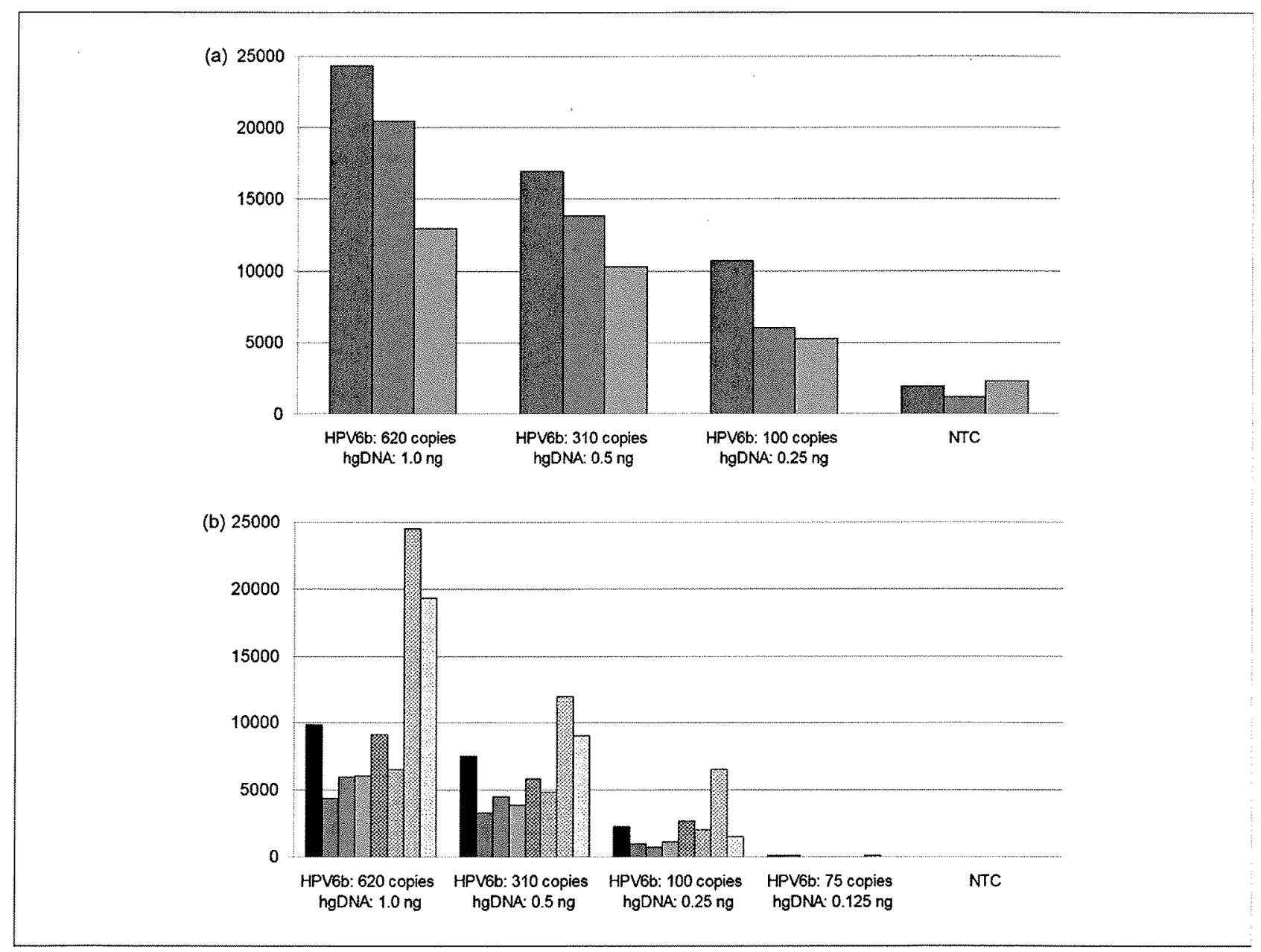

FIGURE 3 Fluorescence data of hybridization of PCR products from integrated amplification, labeling and selective detection of HPV subtype 6b and human $\beta$-globin. All PCRs were performed in the microfluidic channels of a Geniom biochip with primer pairs targeting HPV $6 b$ and human $\beta$-globin with starting template amounts as depicted in the diagram. (a) Fluorescence data of probes specific for human $\beta$-globin. (b) Fluorescence data of probes specific for HPV $6 \mathrm{~b}$. NTC = Non-template control PCR. 


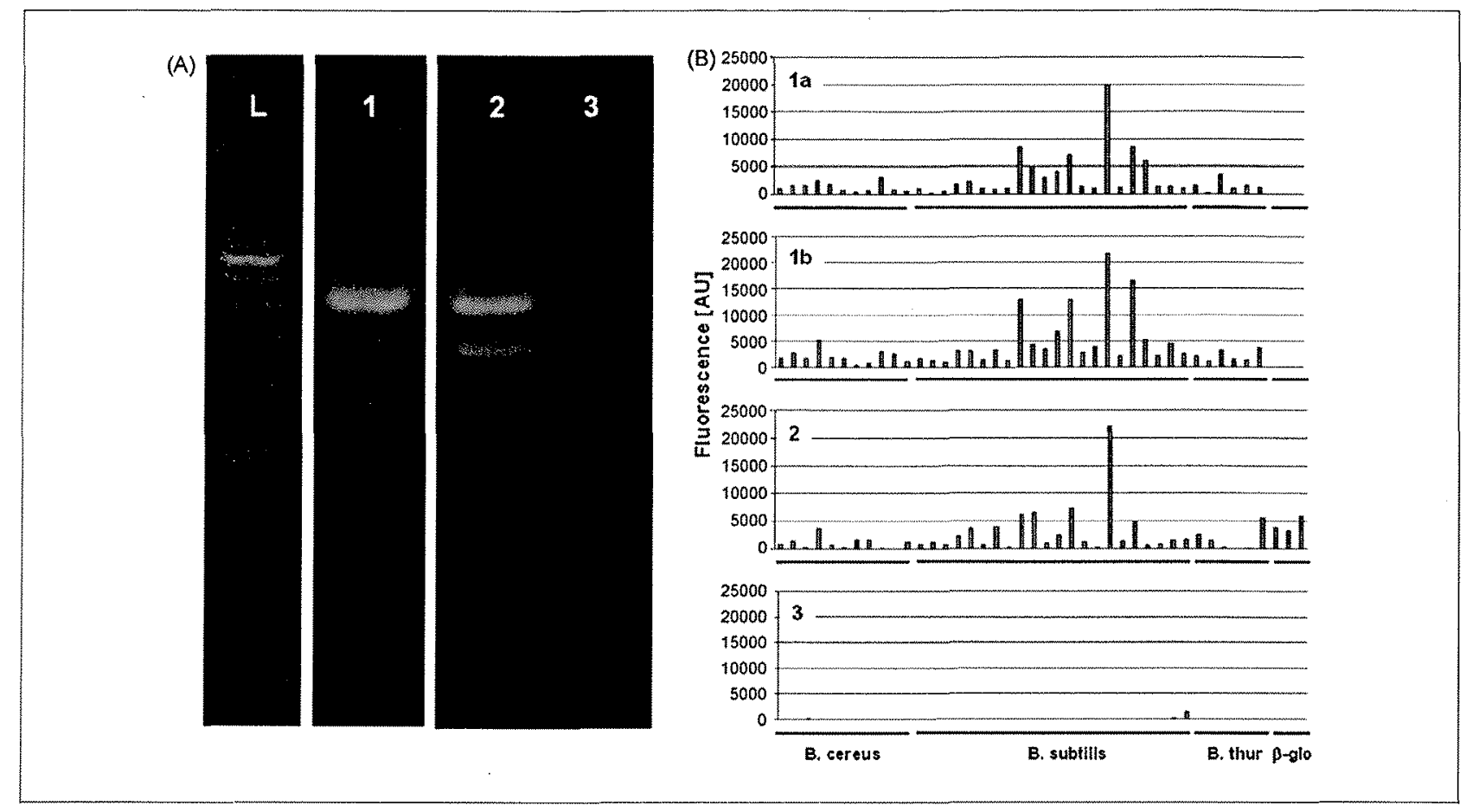

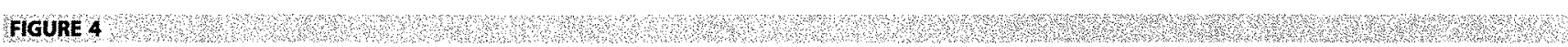

6-Plex PCR targeting different Bacillus species and human $\beta$-globin. (a) Agarose gel analysis of PCRs carried out in PCR tubes using six individual primer pairs and different genomic DNA templates. Expected amplicon lengths for targets are Bacillus cereus: $446 \mathrm{bp}$, Bacillus subtilis: $395 \mathrm{bp}$, Bacillus thuringiensis ser. israelensis: $407 \mathrm{bp}$, human $\beta$-globin: $300 \mathrm{bp} . \mathrm{L}=$ Ladder. 1: PCR conducted in the presence of genomic DNA of Bacillus cereus, Bacillus subtilis and Bacillus thuringiensis ser. israelensis. 2: PCR conducted in the presence of genomic DNA of Bacillus cereus, Bacillus subtilis and Bacillus thuringiensis ser. israelensis and human genomic DNA. 3 : Control PCR conducted in the absence of template DNA. (b) Diagram of fluorescence intensities of different array capture probes obtained after integrated PCR hybridization, fluorescence staining and detection in microchannels of a biochip. Identical PCR mixtures as described in A were used. 1a, b: Two replicates conducted under conditions as used in A, Lane 1. 2, 3: PCRs conducted under conditions as in A, lanes 2 and 3, respectively.

replicates exhibited similar fluorescence intensities (Fig. 4b, 1a,b). No fluorescence was observed for probes specific for human $\beta$ globin. For PCR in the presence of human genomic DNA (Fig. $4 \mathrm{~b}$, 2), fluorescence intensities seemed to differ slightly from PCRs in the absence of human gDNA. This might reflect an impact of the additional product formation on efficiency of PCRs targeting Bacillus species. However, probes correctly indicated the presence of individual Bacillus-related products. Additionally, the presence of the human $\beta$-globin PCR product was clearly indicated by the respective probes. These data show that PCRs involving multiple genomes and up to six primer pairs can be conducted within the microchannels and products can be selectively detected with excellent sensitivity.

In summary, we have developed a fully integrated system to combine all steps of a typical protocol for detection and typing of genomic targets from non-amplified samples. The method allows for semi-quantitative detection and typing of viral, pro- and eukaryotic targets covering different complexities with high sensitivity and selectivity. Owing to the fast cycles of microarray design and testing, the system can be quickly adapted to new genetic variants and provides a theoretical capacity for selective detection of $>600$ target nucleic acids when prototyping is employed in the way presented here. In contrast to previous studies that employ separated amplification and subtyping methods, often involving several further steps for labeling and purification, the highly automated workflow results in low hands-on-time and should be beneficial for reproducibility and contamination risk.

We are currently developing increasingly multiplexed detection arrays for both RNA and DNA targets and evaluate probe collections for detection and subtyping of various viral, as well as proand eukaryotic pathogens that should enable high throughput screening approaches for multiple disease types.

\section{Acknowledgements}

We thank Joanna Grigas for her contributions to the Bacillus studies. This work was funded by the Federal Ministry of Education and Research (BMBF) under contract 0315181.

\section{References}

1 Kaltenboeck, B. and Wang, C. (2005) Advances in real-time PCR: application to clinical laboratory diagnostics. Adv. Clin. Chem. 40, 219-259

2 Wittwer, C.T. et al. (2001) Real-time multiplex PCR assays. Methods 25, 430-442 
5 Solinas, A. et al. (2001) Duplex Scorpion primers in SNP analysis and FRET applications. Nucleic Acids Res. 29, E96

6 Nagai, H. et al. (2001) High-throughput PCR in silicon based microchamber array Biosens. Bioelectron. 16, 1015-1019

7 Nagai, H. et al. (2001) Development of a microchamber array for picoliter PCR. Anal. Chem. 73, 1043-1047

8 Marcus J.S., Anderson, W.F. and Quake, S.R. (2006) Parallel picoliter rt-PCR assays using microfluidics. Anal. Chem. 78, 956-958

9 Kalinina, O. et al. (1997) Nanoliter scale PCR with TaqMan detection. Nucleic Acids Res. $25,1999-2004$

10 Morrison, $T$. et al. (2006) Nanoliter high throughput quantitative PCR. Nucleic Acids Res. 34, e123

11 Brenan, C.J., Roberts, D. and Hurley, J. (2009) Nanoliter high-throughput PCR fo DNA and RNA profiling. Methods Mol. Biol, 496, 161-174

12 Ottesen, E.A. et al. (2006) Microfluidic digital PCR enables multigene analysis of individual environmental bacteria. Science $314,1464-1467$

13 Spurgeon, S.L., Jones, R.C. and Ramakrishnan, R. (2008) High throughput gene expression measurement with real time PCR in a microfluidic dynamic array. PLoS One 3, e1662

14 Weisenberger, DJ. et al. (2008) DNA methylation analysis by digital bisulfite genomic sequencing and digital Methylight. Nucleic Acids Res. 36, 4689 4698

15 Spiro, A. and Lowe, M. (2002) Quantitation of DNA sequences in environmenta PCR products by a multiplexed, bead-based method. Appl. Environ. Microbiol, 68 , 1010-1013

16 Schmitt, M. et al. (2006) Bead-based multiplex genotyping of human papillomaviruses. J. Clin. Microbiol. 44, 504-512

17 Hoheisel, J.D. (2006) Microarray technology: beyond transcript profiling and genotype analysis. Nat. Rev. Genet, 7, 200-210
18 Albrecht, V. et al. (2006) Easy and fast detection and genotyping of high-risk human papillomavirus by dedicated DNA microarrays. J. Virol. Methods 137, 236244

19 Iqbai, J. et al. (2008) Fabrication and evaluation of a sequence-specific oligonucleotide miniarray for molecular genotyping. Indian J. Med. Microbiol. 26, $13-20$

20 Klaassen, C.H. et al. (2004) DNA microarray format for detection and subtyping of human papillomavirus. J. Clin. Microbiol. $42,2152-2160$

21 Uttamchandani, M. et al. (2009) Applications of microarrays in pathogen detection and biodefence. Trends Biotechnol. 27, 53-61

22 Call, D.R. (2005) Challenges and opportunities for pathogen detection using DNA microatrays. Crit. Rev. Microbiol, 31, 91-99

23 Shen, Y. and Wu, B.L. (2009) Microarray-based genomic DNA profiling technologies in clinical molecular diagnostics. Clin. Chtem. 55, 659-669

24 Baum, M. et al. (2003) Validation of a novel, fully integrated and flexible microarray benchtop facility for gene expression profiling. Nucleic Acids Res. 31, e151

25 Chan, S.Y. et al. (1995) Analysis of genomic sequences of 95 papillomavirus types: uniting typing, phylogeny, and taxonomy. J. Virol. 69, 3074-3083

26 Bulkmans, N.W. et al. (2004) POBASCAM, a population-based randomized controlled trial for implementation of high-risk HPV testing in cervical screening: design, methods and baseline data of 44,102 women. Int. J. Cancer 110 , 94-101

27 Chan, P.K. et al. (2006) Biases in human papillomavirus genotype prevalence assessment associated with commonly used consensus primers. Int. J. Cancer 118 , 243-245

28 Gravitt, P.E. et al, (2000) Improved amplification of genital human papillomaviruses. J. Clin. Microbiol. 38, 357-361 